\title{
Bloch-Like Oscillations in a One-Dimensional Lattice with Long-Range Correlated Disorder
}

\author{
F. Domínguez-Adame and V. A. Malyshev* \\ GISC, Departamento de Física de Materiales, Universidad Complutense, E-28040 Madrid, Spain
}

F. A. B. F. de Moura and M. L. Lyra

Departamento de Física, Universidade Federal de Alagoas, Maceió AL 57072-970, Brazil

(Received 13 May 2003; published 6 November 2003)

\begin{abstract}
We study the dynamics of an electron subjected to a uniform electric field within a tight-binding model with long-range-correlated diagonal disorder. The random distribution of site energies is assumed to have a power spectrum $S(k) \sim 1 / k^{\alpha}$ with $\alpha>0$. de Moura and Lyra [Phys. Rev. Lett. 81, 3735 (1998)] predicted that this model supports a phase of delocalized states at the band center, separated from localized states by two mobility edges, provided $\alpha>2$. We find clear signatures of Bloch-like oscillations of an initial Gaussian wave packet between the two mobility edges and determine the bandwidth of extended states, in perfect agreement with the zero-field prediction.
\end{abstract}

DOI: 10.1103/PhysRevLett.91.197402

PACS numbers: 78.30.Ly, 71.30.+h, 72.15.Rn, 73.20.Jc

The single-parameter scaling hypothesis predicts localization of a single quasiparticle in one (1D) and two dimensions with time-reversal symmetry, independently of the disorder strength present in the system [1]. There exist, however, low-dimensional systems that do not obey the single-parameter scaling framework. Thus, the absence of Anderson localization in the presence of spatial short-range correlations in disorder $[2,3]$ was put forward to explain transport properties of semiconductor superlattices with intentional correlated disorder [4]. Further, it was demonstrated that long-range correlated diagonal [5,6] and off-diagonal [7] disorder also acts towards delocalization of 1D quasiparticle states. Furthermore, long-range correlations can result in the emergence of a phase of extended states in the thermodynamic limit. This phase appears at the band center and is separated from localized states by two mobility edges [5]. This theoretical prediction was experimentally validated by measuring microwave transmission spectra of a single-mode waveguide with inserted correlated scatterers [8]. In the case of short-range correlated disorder, the delocalized phase does not appear: The number of delocalized states increases proportionally to the square root of the system size, and thus this phase has zero measure in the thermodynamic limit.

In this Letter, we focus on the dynamical properties of an electron in a system with long-range correlated diagonal disorder. Interplay between the delocalization effect, preserved by the long-range correlated disorder, and the dynamic localization, caused by an electric field acting on the system, is of our interest. We compute the behavior of an initial Gaussian wave packet in the presence of a uniform electric field solving numerically the 1D time-dependent Schrödinger equation for the complete Hamiltonian. We found clear signatures of Bloch-like oscillations [9] of the wave packet between the two mobility edges of the delocalized phase of states. The am- plitude of the oscillatory motion of the centroid allows us to determine the bandwidth of the delocalized phase.

We consider a tight-binding Hamiltonian with longrange-correlated diagonal disorder and an external dc electric field on a regular 1D open lattice of spacing $a$ [10]

$$
\begin{aligned}
\mathcal{H}= & \sum_{n=1}^{N}\left(\tilde{\varepsilon}_{n}-e \mathcal{F} a n\right)|n\rangle\langle n| \\
& -J \sum_{n=1}^{N-1}(|n\rangle\langle n+1|+| n+1\rangle\langle n|),
\end{aligned}
$$

where $|n\rangle$ is a Wannier state localized at site $n$ with energy $\tilde{\varepsilon}_{n}, \mathcal{F}$ is the external uniform electric field, and $N$ is even. The intersite coupling is restricted to nearest neighbors and assumed to be uniform over the entire lattice with $J>0$. In terms of the Wannier amplitudes $\psi_{n}(t)=$ $\langle n \mid \Psi(t)\rangle$, the Schrödinger equation reads [11]

$$
i \dot{\psi}_{n}=\left(\varepsilon_{n}-F n\right) \psi_{n}-\psi_{n+1}-\psi_{n-1},
$$

where we introduced the dimensionless parameters $\varepsilon_{n}=$ $\tilde{\varepsilon}_{n} / J, F=e \mathcal{F} a / J$, and time is expressed in units of $\hbar / J$.

The source of disorder is the stochastic fluctuations of energies $\epsilon_{n}$, which we are going to consider as being longrange correlated. One of the simplest ways to numerically generate a power-law correlated sequence of on-site potentials $\varepsilon_{n}$ is to write its Fourier decomposition as follows [5]:

$$
\varepsilon_{n}=C(\alpha) \sum_{k=1}^{N / 2} \frac{1}{k^{\alpha / 2}} \cos \left(\frac{2 \pi n k}{N}+\phi_{k}\right) .
$$

Here, $\phi_{k}$ are $N / 2$ independent random phases uniformly distributed within the interval $[0,2 \pi]$, and $C(\alpha)$ is a normalization constant. We will normalize the energy sequence to have $\left\langle\varepsilon_{n}\right\rangle=0$ and $\left\langle\varepsilon_{n}^{2}\right\rangle=1$, where $\langle\cdots\rangle$ indicates average over the random phases $\phi_{k}$. The 
long-range nature of the potential correlations results from the power-law dependence of the amplitudes on the wave vector characterizing each Fourier component. Several stochastic processes in nature are known to generate long-range correlated random sequences which have no characteristic scale, for example, in the nucleotide sequence of DNA molecules [12]. The relevance of the underlying long-range correlations for the electronic transport in DNA has been recently discussed in Ref. [13]. Furthermore, interface roughness appearing during growth often displays height-height correlations with power-law spectra [14]; thus, the subsequent random potential arising from the rough interface would be long-range correlated. Recently, transport properties of systems with long-range correlated disorder was explored, both theoretically and experimentally, in the design of devices for filtering of electrical and optical signals [15].

Having introduced the model of disorder, we can solve (2) numerically by means of an implicit integration algorithm [16]. The initial condition is set to a Gaussian wave packet of width $\sigma$ and centered at $n_{0}=N / 2$ :

$$
\psi_{n}(0)=A(\sigma) \exp \left[-\left(n-n_{0}\right)^{2} / 4 \sigma^{2}\right],
$$

$A(\sigma)$ being the normalization factor. Once Eq. (2) is solved for the initial condition (4), we compute the mean position of the wave packet (centroid):

$$
x(t)=\sum_{n=1}^{N}\left(n-n_{0}\right)\left|\psi_{n}(t)\right|^{2} .
$$

It is to be noticed that all eigenstates of the Hamiltonian (1) contribute to $x(t)$.

It is well known that a uniform electric field applied to a periodic lattice causes the dynamic localization of the initially extended states of an electron and gives rise to an oscillatory behavior of the electron wave packet, the socalled Bloch oscillations [9]. The length of the segment over which the electron oscillates (twice the amplitude) and the period of these oscillations are estimated from semiclassical arguments to be (in dimensionless units) $L_{F}=W / F$ and $\tau_{B}=2 \pi / F$, respectively (e.g., see Ref. [17]), where $W$ is the width of the Bloch band in units of the coupling integral $J$ ( $W=4$ in our case). Notice that this approach requires slowly varying wave functions to be valid, implying that $L_{F}>1$. A similar condition was pointed out in Ref. [18]. The question we aim to clarify is to what extent the corresponding phenomenology will be valid if long-range correlated disorder, which allows for a delocalized phase, is present; in other words, whether the biased phase of extended states bounded by two mobility edges behaves similarly to a biased Bloch band. If so, it provides us with a method to measure the energy width of the delocalized phase $W^{\prime}$ from the relationship $L_{F}^{\prime}=W^{\prime} / F$, where $L_{F}^{\prime}$ is twice the amplitude of the oscillatory motion of the centroid. Below, we present a numerical proof of this conjecture.
In Fig. 1, we show the asymptotic dynamics $\left(t \gg \tau_{B}\right)$ of the Wannier amplitudes $\psi_{n}(t)$ of an initial Gaussian wave packet $\psi_{n}(t)(\sigma=10$ at $t=0$ and $F=0.1)$ calculated for two values of the exponent $\alpha, 0.5$ (the phase of delocalized states is absent in the unbiased system) and 3.0 (the phase of delocalized states is present in the unbiased system). At $\alpha=0.5$ [see Fig. 1(a)], the disorder is almost uncorrelated and the system is similar to the standard Anderson model, with no signatures of Bloch oscillations. On the contrary, the plot in Fig. 1(b) calculated for $\alpha=3.0$ shows an oscillating in time pattern. This result suggests that Bloch oscillations can take place even in the presence of disorder.

The time-domain evolution of the centroid, $x(t)$, provides more detailed information about what is happening. In Fig. 2, one can see that there are no signatures of Bloch oscillations for $\alpha=0.5$ (dotted line), at least for a moderate field amplitude. Oscillations, which are present at the very beginning, achieve in a short time a weakly fluctuating (stationary in average) value. This is a clear indication of the existence of disorder-induced decoherence effects, as is expected for the localized regime. On the contrary, at $\alpha=3.0$ the centroid displays an
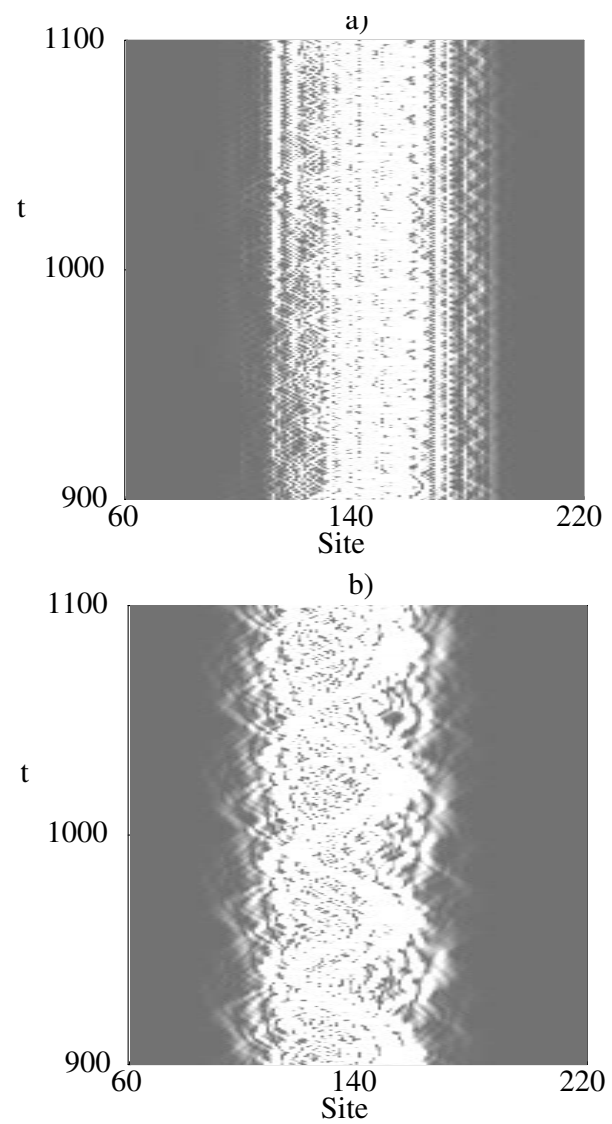

FIG. 1. Asymptotic dynamics $\left(t \gg \tau_{B}\right)$ of the Wannier amplitudes $\psi_{n}(t)$ of a biased wave packet $(\sigma=10$ at $t=0$ and $F=0.1$ ) in a lattice with $N=300$ sites, for (a) $\alpha=0.5$ and (b) $\alpha=3.0$. Light and dark regions indicate higher and lower probability amplitudes, respectively. 


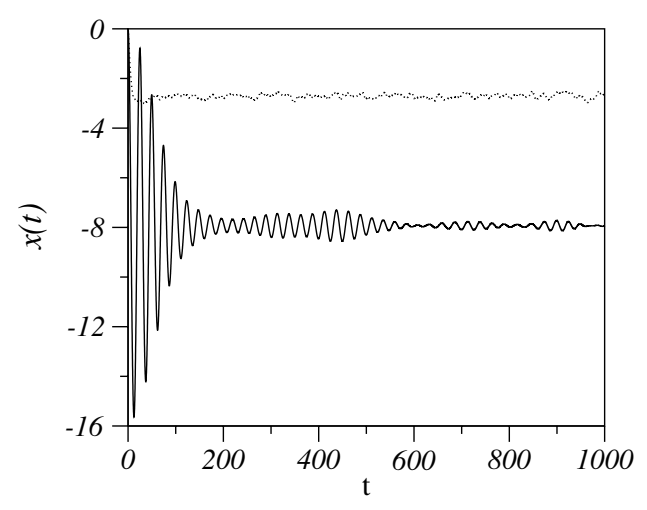

FIG. 2. Time-domain dynamics of the centroid of a biased wave packet $(\sigma=10$ at $t=0$ and $F=0.25$ ) in a lattice with $N=500$ sites. Dotted lines correspond to $\alpha=0.5$ and solid lines to $\alpha=3.0$.

oscillatory, amplitude-modulated pattern after an initial transient, where the amplitude of the oscillations is reduced (solid line). This oscillatory displacement of the wave packet is not accompanied by in-phase oscillation of its width. In other words, breather modes are absent for the specified initial condition.

Valuable information can be extracted from a detailed inspection of the centroid dynamics. First, we notice that the period of the oscillations is a well-defined quantity, as is seen from Fig. 3. Furthermore, within the numerical uncertainty, its value is equal to $\tau_{B}=2 \pi / F$, being the period of Bloch oscillations in a homogeneous lattice. At the initial stage of the motion, the spatial region within which the wave packet oscillates is roughly given by $4 / F$, as for an ideal Bloch band. Disorder results in a relatively fast decrease of the oscillation amplitude. After this transient stage, oscillations are not damped further and remains amplitude modulated with an envelope which depends on the realization of disorder. Dotted lines in Fig. 3 bound the spatial region within which the wave packet oscillates for a long time. On average, the width of this region $L_{F}^{\prime}$ is found to be $L_{F}^{\prime} \sim W^{\prime} / F$, where $W^{\prime}$ is independent of the applied field $F$. From the data in Fig. 3, we obtain $W^{\prime} \sim 1$. This value agrees remarkably well with the width of the band of extended states reported in Ref. [5] for $\alpha=3.0$. Thus, we arrive at the main conclusion of this work; namely, there exist clear signatures of Bloch-like oscillations of a biased Gaussian wave packet between the two mobility edges.

To provide further confirmation of this claim, we calculated numerically the Fourier transform of the centroid, $\tilde{x}(\omega)$, as shown in Fig. 4. Results were obtained by averaging over 1000 realizations of disorder. For $\alpha=0.5$, the Fourier transform $\tilde{x}(\omega)$ is rather broad, suggesting that $x(t)$ is similar to a white noise signal. On the contrary, for $\alpha=3.0$ the Fourier transform $\tilde{x}(\omega)$ shows a well-defined, narrow peak at about $\omega=F$, despite averaging. The variance of $\tilde{x}(\omega), \sigma_{\omega}^{2}$, displays a rather different trend below and above $\alpha=2.0$, as seen in the inset of Fig. 4. For $\alpha<2.0$, the Fourier transform is broad, but for
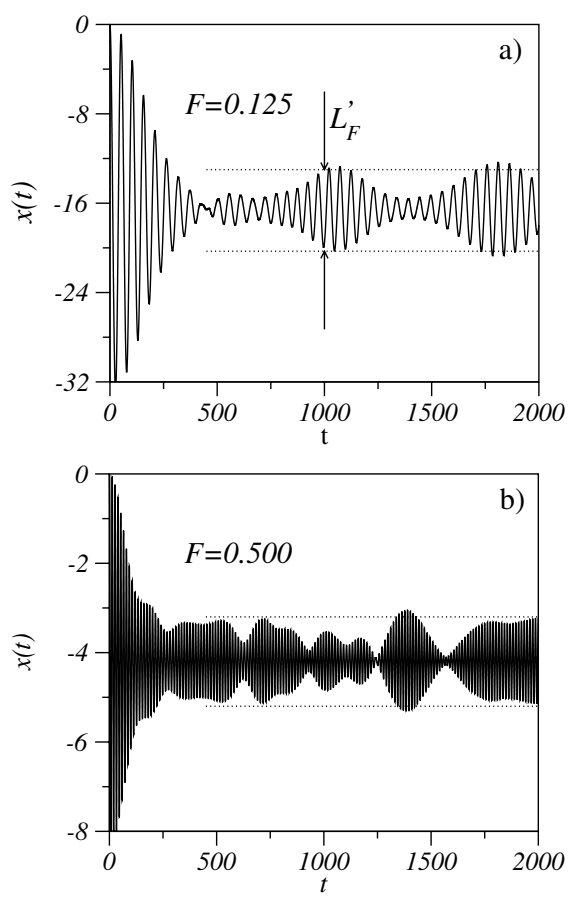

FIG. 3. Time-domain dynamics of the centroid of a biased wave packet $(\sigma=10$ at $t=0$ ) in a lattice with $N=1000$ when $\alpha=3.0$, for (a) $F=0.125$ and (b) $F=0.5$. Dotted lines bound a region of size $L_{F}^{\prime}$ within which the wave packet oscillates for a long time.

$\alpha>2.0$ the width of the Fourier transform is rather small and independent of the electric field. The onset for the appearance of Bloch oscillations $\alpha=2$ (see the inset of Fig. 4) is in excellent agreement with the value obtained in Ref. [5] for the occurrence of the phase of extended states.

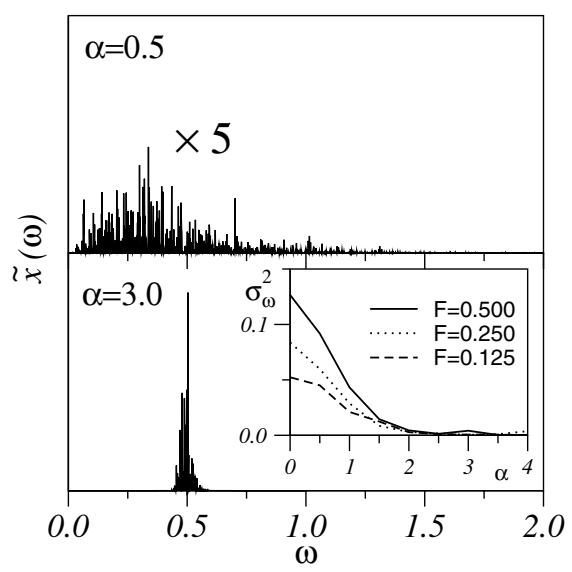

FIG. 4. Fourier transform of the centroid of a biased wave packet ( $\sigma=10$ at $t=0$ and $F=0.5$ ) in a lattice with $N=500$ sites, for $\alpha=0.5$ (upper panel; notice the magnification factor) and $\alpha=3.0$ (lower panel). The inset shows the variance $\sigma_{\omega}^{2}$ of $\tilde{x}(\omega)$ as a function of $\alpha$ for different values of the applied electric field. Results were obtained by averaging over 1000 realizations of the disorder. 


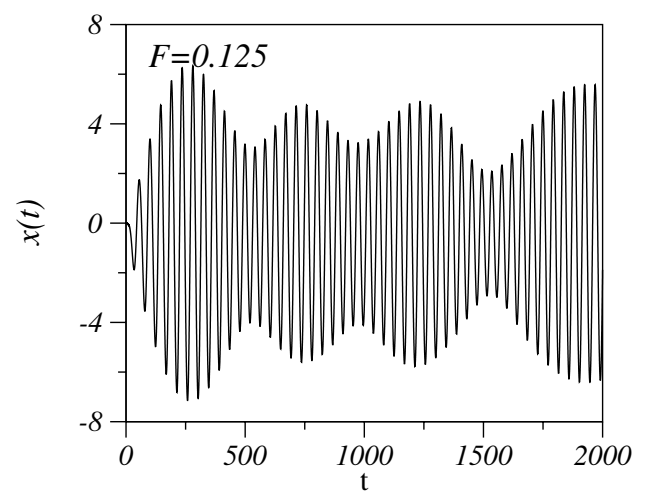

FIG. 5. Time-domain dynamics of the centroid of a biased wave packet, with initial condition $\psi_{n}(0)=\delta_{n n_{0}}$, in a lattice with $N=1000$ sites when $\alpha=3.0$, for $F=0.125$.

The above results for $F \neq 0$ were obtained with a relatively broad wave packet $(\sigma \gg 1)$ with initial velocity equal to zero. Consequently, its Fourier components span a very narrow set of states at the bottom of the band. We also performed a study for initial moving wave packets located initially deep in the band. In short, in the very beginning the wave packet explores the entire band in either case and oscillates within a spatial region of size $4 / F$. On increasing time, the amplitude of $x(t)$ is always reduced to a region of size $L_{F}^{\prime}=W^{\prime} / F$, where $W^{\prime}$ is the width of the delocalized phase. This picture changes slightly for narrower initial wave packets, for which the large-amplitude transient is absent. Figure 5 shows the centroid dynamics of an initial Kronecker $\delta$ wave packet at $\alpha=3.0$ and $F=0.125$. The extent of the spatial region within which the wave packet asymptotically oscillates is again $L_{F}^{\prime}=W^{\prime} / F$. Thus, we can confidently state that Bloch-like oscillations are rather insensitive to initial conditions.

We studied a biased random tight-binding model where the on-site disorder is long-range correlated with the power spectrum $S(k) \sim 1 / k^{\alpha}, \alpha>0$. The unbiased model supports a phase of delocalized states in the center of the band provided $\alpha>2$ [5]. We found clear signatures of Bloch-like oscillations for $\alpha>2$ and their absence for $\alpha<2$. The period of the oscillations agrees well with the period in an ideal Bloch band. Thus, the amplitude of the oscillations provides a direct way to estimate the energy difference between the two mobility edges of the delocalized phase. We found that this energy difference is in perfect agreement with the results reported in Ref. [5]. This finding opens the possibility to experimentally measure the bandwidth of the delocalized phase. Actual technological advances made it possible to monitor the amplitude of Bloch oscillations in uniform semiconductor superlattices [19]. Recently, intentionally disordered superlattices were used to demonstrate the absence of localization in short-range correlated disordered systems
[4]. Thus, we conjecture that intentionally disordered superlattices with long-range correlated disorder would allow for a clearcut validation of the present results. In addition, metallic rings threaded by magnetic fields linearly rising in time also display Bloch oscillations [18], thus opening new experimental ways to test our predictions.

V. A. M. acknowledges support from NATO. Work at Madrid was supported by DGI-MCyT (MAT20000734). Work at Brazil was supported by $\mathrm{CNPq}$ and CAPES (Brazilian research agencies) and FAPEAL (Alagoas State agency).

*On leave from "S.I. Vavilov State Optical Institute," Birzhevaya Linia 12, 199034 Saint-Petersburg, Russia.

[1] E. Abrahams, P.W. Anderson, D. C. Licciardello, and T.V. Ramakrishnan, Phys. Rev. Lett. 42, 673 (1979).

[2] J. C. Flores, J. Phys. Condens. Matter 1, 8471 (1989).

[3] D. H. Dunlap, H.-L. Wu, and P.W. Phillips, Phys. Rev. Lett. 65, 88 (1990).

[4] V. Bellani, E. Diez, R. Hey, L. Toni, L. Tarricone, G. B. Parravicini, F. Domínguez-Adame, and R. GómezAlcalá, Phys. Rev. Lett. 82, 2159 (1999).

[5] F. A. B. F. de Moura and M. L. Lyra, Phys. Rev. Lett. 81, 3735 (1998).

[6] F. M. Izrailev and A. A. Krokhin, Phys. Rev. Lett. 82, 4062 (1999).

[7] F. A. B. F. de Moura and M. L. Lyra, Physica (Amsterdam) 266A, 465 (1999).

[8] U. Kuhl, F. M. Izrailev, A. A. Krokhin, and H.-J. Stöckmann, Appl. Phys. Lett. 77, 633 (2000).

[9] F. Bloch, Z. Phys. 52, 555 (1928).

[10] D. H. Dunlap and V. M. Kenkre, Phys. Rev. B 34, 3625 (1986).

[11] H. N. Nazareno and P. E. de Brito, Phys. Rev. B 60, 4629 (1999).

[12] C.-K. Peng, S.V. Buldyrev, A. L. Goldberger, S. Havlin, F. Sciortino, M. Simons, and H. E. Stanley, Nature (London) 356, 168 (1992).

[13] P. Carpena, P. Bernaola-Galván, P. Ch. Ivanov, and H. E. Stanley, Nature (London) 418, 955 (2002).

[14] A.-L. Barabási and H. E. Stanley, Fractal Concepts in Surface Growth (Cambridge University Press, Cambridge, England, 1995).

[15] A. Krokhin, F. Izrailev, U. Kuhl, H.-J. Stöckmann, and S. E. Ulloa, Physica (Amsterdam) 13E, 695 (2002).

[16] W. H. Press, B. P. Flannery, S. A. Teukolsky, and W.T. Wetterling, Numerical Recipes: The Art of Scientific Computing (Cambridge University Press, New York, 1986), pp. 656-663.

[17] N.W. Ashcroft and N. D. Mermin, Solid State Physics (Saunders College Publishers, New York, 1976), p. 213.

[18] L. Arrachea, Phys. Rev. B 66, 045315 (2002).

[19] V. G. Lyssenko, G. Valusis, F. Loser, T. Hasche, K. Leo, M. M. Dignam, and K. Kohler, Phys. Rev. Lett. 79, 301 (1997). 